08

\title{
Дисперсия масштабно-инвариантных функций распределения по размерам
}

\author{
() В.Г. Дубровский
}

Санкт-Петербургский академический университет

Физико-технический институт им. А.Ф. Иофффе РАН, Санкт-Петербург Санкт-Петербургский национальный исследовательский университет информационных технологий, механики и оптики

E-mail: Dubrovskii@mail.ioffe.ru

\section{Поступило в Редакцию 24 ноября 2016 г.}

Показано, что функция распределения по размерам наноструктур, удовлетворяющая свойству масштабной инвариантности (скейлинга), всегда имеет дисперсию, пропорциональную квадрату среднего размера. Проведен теоретический анализ формы и дисперсии одного двухпараметрического масштабноинвариантного распределения, описывающего рост гомогенных или гетерогенных нанобъектов с линейными по размеру скоростями захвата мономеров. Показан переход от гауссова к более широкому и несимметричному распределению при уменьшении вероятности нуклеации.

DOI: 10.21883/PJTF.2017.09.44570.16584

Сформулированное впервые Vicsek и Family в работе [1] свойство масштабной инвариантности (МИ), или скейлинга, функции распределения по размерам (ФР) наблюдается для некоторых двумерных поверхностных островков на ранних стадиях эпитаксиального роста, предшествуюших коалесценции [2-4]. Для этого необходимо, чтобы коэффициент диффузии адатомов (подвижных мономеров) был много 
больше скорости их осаждения на подложку. МИ ФР имеют вид [1-4]

$$
n(s,\langle s\rangle, \Theta)=\frac{\Theta}{\langle s\rangle^{2}} F(x), \quad x=\frac{s}{\langle s\rangle},
$$

где $s$ - число мономеров в островке („размер“, пропорциональный площади поверхности, занимаемой островком), $\langle s\rangle-$ средний размер островков и $\Theta$ - степень заполнения поверхности подложки островками. Скейлинговая функция $(С \Phi) F(x)$ в правой части приведенного выражения должна быть универсальной, т.е. не зависеть ни от $\Theta$, ни от $\langle s\rangle$. Таким образом, измеренные в различные моменты времени (и соответствующие различным $\Theta$ и $\langle s\rangle)$ ФР, перестроенные в масштабноинвариантных переменных, идентичны. Данное свойство имеет большое значение для физических свойств ансамблей наноструктур, получаемых при усреднении различных величин с МИ ФР. При высоких значениях коэфициента диффузии в сравнении со скоростью осаждения осуществляется режим полной конденсации тонких пленок [2-4], в котором концентрация мономеров быстро стремится к нулю. Тогда поверхностная плотность островков $N$ примерно равна $\Theta /\langle s\rangle$ и для нормированной на плотность $Ф$ Р $f=n / N$ справедливо более простое выражение

$$
f(s,\langle s\rangle)=\frac{F(x)}{\langle s\rangle},
$$

которое и будет использоваться в дальнейшем. Задача данной работы состоит в исследовании формы и ширины ФР по размерам, удовлетворяющих гипотезе масштабной инвариантности.

Из определения поверхностной плотности островков и их среднего размера из (1) получаем два известных свойства нормировки, требуемые от любой СФ

$$
\int_{0}^{\infty} d x F(x)=\int_{0}^{\infty} d x x F(x)=1 .
$$

Для дисперсии ФР

$$
\sigma=\left\langle(s-\langle s\rangle)^{2}\right\rangle=\left\langle s^{2}\right\rangle-\langle s\rangle^{2} .
$$

Из (1) следует

$$
\sigma=\left[\int_{0}^{\infty} d x x^{2} F(x)-1\right]\langle s\rangle^{2} .
$$


Данное выражение является первым результатом работы и показывает, что любая МИ ФР обязана быть достаточно широкой, с дисперсией, пропорциональной квадрату среднего размера. Известно, например, что пуассоновское распределение и его гауссова континуальная асимптотика имеют дисперсию, равную среднему размеру, и поэтому не могут быть масштабированы в аналитическую СФ. С этим фактом связаны неаналитические СФ, обсуждаемые в работах [5-7] для моделей необратимого роста, в которых „среднеполевые“ скорости роста островков пропорциональны $s^{\beta}$ со степенным индексом $0 \leq \beta<1$. Тем более не удовлетворяют свойству МИ узкие ФР, обычные в теории нуклеации с распадом частиц $[8,9]$.

В скейлинговом пределе больших подвижностей становится существенной конкуренция соседних островков за диффузионный поток адатомов, что приводит к линейной зависимости скоростей роста от $s$ при достаточно больших $s[3,4]$. Если предположить, что данная зависимость справедлива абсолютно для всех, а не только для больших $s$, полученная при некоторых дополнительных предположениях в работе [10] СФ имеет вид

$$
F(x)=\frac{b c^{b}}{\Gamma(a+1)} x^{b-1} \Gamma(c x, a-b+1), \quad c=\frac{(a+1) b}{(b+1)} .
$$

Здесь $\Gamma(y, q)$ и $\Gamma(q)$ - верхняя неполная гамма-функция и полная гамма-функция, определенные согласно

$$
\Gamma(y, q)=\int_{y}^{\infty} d t t^{q-1} e^{-t}, \quad \Gamma(q) \equiv \Gamma(0, q) .
$$

Поскольку константа $c$ однозначно определяется через $a$ и $b$, СФ $F(x)$ в (4) зависит от двух положительных (а в остальном - произвольных) параметров $a$ и $b$, физический смысл которых может быть различным. Для гомогенного роста двумерных островков данные параметры расшифрованы в [10]. Легко проверить, что СФ (4) удовлетворяет условиям нормировки (2) при любых $a$ и $b$.

В самое последнее время модели гетерогенного необратимого роста $[11,12]$ стали применяться для моделирования ФР по длине III-V нитевидных нанокристаллов (ННК). Если III-V ННК растут из катализаторов $\mathrm{Au}[13,14]$ по механизму диффузионного роста в условиях,

Письма в ЖТФ, 2017, том 43, вып. 9 
обогащенных по группе V [15], и длина ННК менее диффузионной длины адатомов металлов III группы на боковой поверхности ННК [12], схема роста может быть представлена в виде

$$
\begin{aligned}
A_{1}+B & \stackrel{b}{\rightarrow} A_{1} B+A_{1} \stackrel{a+1}{\longrightarrow} A_{2} B+A_{1} \rightarrow \ldots \\
& \rightarrow A_{s} B+A_{1} \stackrel{a+s}{\longrightarrow} A_{s+1} B+A_{1} \rightarrow \ldots
\end{aligned}
$$

Здесь $B$ обозначает каплю катализатора, из которой происходит рост ННК, $A_{1}$ - монослой ННК (мономер), добавление которого на вершину ННК приводит к вертикальному росту последнего, $A_{s} B$ с $s=1,2,3 \ldots$. есть ННК, состоящий из монослоев III-V полупроводника и капли катализатора на вершине. Константа $b$ есть, очевидно, скорость нуклеации первого монослоя ННК из капли. Скорость удлинения ННК с $s \geq 1$ равна $a+s$ и содержит вклады как от диффузии адатомов на вершину ННК, так и от прямого потока в каплю. В работе [12] рассматривался упрощенный случай $b=a$, при этом отсутствует задержка нуклеации первого монослоя ННК по отношению к верхним. Можно показать (этот нетривиальный результат будет строго выведен в отдельном сообщении), что СФ (4) описывает континуальную аппроксимацию точного решения дискретных уравнений баланса для ФР по длине ННК при $s \gg 1$, с экспоненциально зависящей от времени $\tau$ средней длиной $\langle s\rangle=c\left(e^{\tau}-1\right) \cong c e^{\tau}$. Никаких дополнительных предположений о скорости диффузии адатомов при этом не требуется. Поэтому дальнейшие результаты будут формулироваться для гетерогенной схемы роста (5) и в терминологии ННК. Отметим, однако, что схема (5) может представлять интерес и для других нанообъектов, например, самоиндуцированных квантовых точек и наноигл на рассогласованных подложках $[16,17]$.

Второй задачей работы является исследование дисперсии и формы СФ (4) при различных $b$ и больших $a \gg 1$ (обоснование этого сильного неравенства для ННК дано в [12]). Применяя общую формулу (3) для СФ (4), получаем

$$
\sigma=\frac{[a+2+b(b+2)]}{(a+1) b(b+2)}\langle s\rangle^{2} .
$$

Очевидно, при $b=a$ СФ (4) сводится к

$$
F(x)=\frac{a^{a}}{\Gamma(a)} x^{a-1} e^{-a x},
$$

Письма в ЖТФ, 2017, том 43, вып. 9 
что при $a \gg 1$ эквивалентно симметричному гауссиану

$$
F(x)=\sqrt{\frac{a}{2 \pi}} \exp \left[-\frac{a}{2}(x-1)^{2}\right]
$$

с дисперсией

$$
\sigma=\frac{\langle s\rangle^{2}}{a}
$$

Этот результат, полученный в работе [12], совпадает с (6) при $b=a$.

Исследуем теперь случай медленной нуклеации ННК, когда $a \gg 1$ и $a \gg b$. Асимптотика (4) при достаточно малых $b$ дает „треугольную“ СФ

$$
F(x)= \begin{cases}\frac{b^{b+1}}{(b+1)^{b}} x^{b-1}, & x<\frac{b+1}{b}, \\ 0, & x>\frac{b+1}{b} .\end{cases}
$$

Дисперсия данной ФР равна

$$
\sigma=\frac{\langle s\rangle^{2}}{b(b+2)} .
$$

Аналогичный результат для дисперсии следует из общей формулы (6) при $a \rightarrow \infty$ и конечном $b$. Таким образом, континуальная СФ при $b=a \gg 1$ дает симметричный гауссиан, в то время как задержка нуклеации ННК приводит к гораздо более широкому треугольному распределению с затянутым левым хвостом. При $b<1$ СФ монотонно убывает и имеет интегрируемую особенность в нуле. При $b>1$ СФ является распределением с максимумом, которое становится все более узким и симметричным при увеличении $b$.

На рисунке показан переход от симметричной ФР в виде гауссиана при $b=a=100$ к более широким и несимметричным ФР при уменьшении вероятности нуклеации $b$. Пунктирные линии при $b=0.5,1$ и 3 соответствуют треугольным детерминированным СФ (10) и хорошо описывают затянутые левые хвосты точных ФР. Однако их крутые передние хвосты размываются за счет кинетических флуктуаций $[7,8]$. Как следствие, детерминированные СФ становятся неточными в области больших размеров, и расхождение с точными ФР увеличивается с ростом $b$.

Письма в ЖТФ, 2017, том 43, вып. 9 


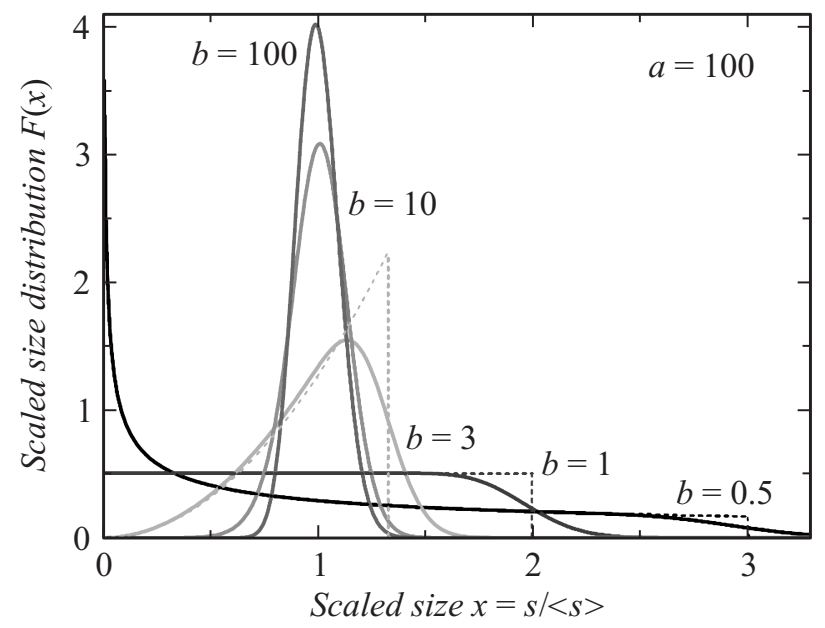

Двухпараметрические СФ, определяемые формулой (4) при $a=100$ и различных $b$. Форма ФР становится более размытой и асимметричной при уменьшении $b$. Соответствующая данным ФР дисперсия увеличивается от $\langle s\rangle^{2} / a$ при $b=a$ до $\langle s\rangle^{2} /[b(b+2)]$ при $b \ll a$. Пунктирные линии соответствуют детерминированным треугольным распределениям (9).

В заключение, нами показано, что любая МИ ФР имеет дисперсию, пропорциональную квадрату среднего размера. Коэффициент пропорциональности, однако, может отличаться на порядки величины и соответствовать абсолютно различным по форме распределениям. Это было продемонстрировано на примере двухпараметрической СФ, пригодной для описания ФР по длине III-V ННК, растущих по диффузионному механизму при наличии задержки нуклеации ННК. При $b=a \gg 1$ данная $Ф Р$ имеет вид гауссиана, но расплывается и становится все более несимметричной при уменьшении $b$. Проведенное рассмотрение позволяет сформулировать некоторые общие рецепты для описания и интерпретации экспериментальных ФР. Прежде всего, необходимо перестроить ФР в масштабированных переменных и проверить, что вид полученной СФ не зависит от времени. Масштабированные $Ф Р$, не отвечающие гипотезе МИ, обычно сужаются при увеличении времени роста (или среднего размера). Если СФ инвариантна, ее симметрия обычно означает отсутствие препятствий к гетерогенной нуклеации.

Письма в ЖТФ, 2017, том 43, вып. 9 
Напротив, расплывание СФ и образование затянутого хвоста в области малых размеров свидетельствуют о задержках нуклеации, которые могут объясняться различными причинами (медленным материальным транспортом или энергетикой системы).

Данная работа была поддержана грантами Российского фонда фундаментальных исследований № 15-52-78057, 16-02-00134 и 16-29-03129.

\section{Список литературы}

[1] Vicsek T., Family F. // Phys. Rev. Lett. 1984. V. 52. P. 1669.

[2] Brune H. // Surf. Sci. Rep. 1998. V. 31. P. 121.

[3] Evans J.W., Thiel P.A., Bartelt M.C. // Surf. Sci. Rep. 2006. V. 61. P. 1.

[4] Einax M., Dieterich W., Maass P. // Rev. Mod. Phys. 2013. V. 85. P. 921.

[5] Bartelt M.C., Evans J.W. // Phys. Rev. B. 1992. V. 46. P. 12675.

[6] Vvedensky D.D. // Phys. Rev. B. 2000. V. 62. P. 15435.

[7] Dubrovskii V.G., Sibirev N.V. // Phys. Rev. B. 2014. V. 89. P. 054305.

[8] Dubrovskii V.G. // J. Chem. Phys. 2009. V. 131. P. 164514.

[9] Kukushkin S.A., Osipov A.V. // Prog. Surf. Sci. 1996. V. 51. P. 1.

[10] Dubrovskii V.G., Sibirev N.V. // Phys. Rev. E. 2015. V. 91. P. 042408.

[11] Dubrovskii V.G. // Phys. Rev. B. 2013. V. 87. P. 195426.

[12] Dubrovskii V.G., Berdnikov Y., Schmidtbauer J. et al. // Cryst. Growth Des. 2016. V. 16. P. 2167.

[13] Wagner R.S., Ellis W.C. // Appl. Phys. Lett. 1964. V. 4. P. 89.

[14] Дубровский В.Г., Сибирев Н.В., Цырлин Г.Э. // Письма в ЖТФ. 2004. Т. 30. B. 16. C. 41 .

[15] Dubrovskii V.G. // Appl. Phys. Lett. 2014. V. 104. P. 053110.

[16] Ng K.W., Ko W.S., Tran T.T.D. et al. // ACS Nano. 2013. V. 7. P. 100.

[17] Zhang X., Dubrovskii V.G., Sibirev N.V. et al. // Cryst. Growth Des. 2011. V. 11. P. 5441. 